\title{
GERMINAÇÃO E CRESCIMENTO DE Caesalpinia ferrea MART. EX TUL. EM DIFERENTES SUBSTRATOS ${ }^{1}$
}

\author{
Silvana de Paula Quintao Scalon², Tiara Kesli Conticelli Teodósio², José Oscar Novelino², \\ Camila Kissmann ${ }^{3}$ e Leandro Henrique de Sousa Mota ${ }^{4}$
}

\begin{abstract}
RESUMO - A produção de mudas de espécies nativas para plantios comerciais e para recuperação de áreas degradadas faz com que haja grande procura por tecnologia que reduza os custos de estabelecimento dessas espécies, como substratos alternativos. Com o objetivo de proporcionar melhor emergência e crescimento inicial das plantas de Caesalpinia ferrea Mart. ex Tul., foram testados diferentes substratos, utilizando-se Latossolo Vermelho Distroférrico, textura argilosa - T, misturado com areia - A $\left(0,5 \mathrm{dm}^{3} \mathrm{de}\right.$ terra/ 0,5 $\mathrm{dm}^{3}$ de areia), acrescentando diferentes proporções de adubo orgânico organosuper ${ }^{\circledR}(\mathrm{AO})$ e adubo químico Yoorin ${ }^{\circledR}$ (AQ), constituindo os seguintes substratos: 1) $\mathrm{T}+\mathrm{A}$ (testemunha); 2$) \mathrm{T}+\mathrm{A}+\mathrm{AO}\left(9,86 \mathrm{~g} \mathrm{dm}^{-3}\right)$; 3) $\mathrm{T}+\mathrm{A}+\mathrm{AO}$ $\left(14,69 \mathrm{~g} \mathrm{dm}^{-3}\right)$; 4) T+A $+\mathrm{AO}\left(19,46 \mathrm{~g} \mathrm{dm}^{-3}\right)$, 5) A+T+AQ $\left(8,4 \mathrm{~g} \mathrm{dm}^{-3}\right)$. A composição terra + areia $+19,46 \mathrm{~g} \mathrm{dm}^{-3} \mathrm{do}^{-}$ adubo orgânico proporcionou melhor emergência das plântulas e maior Índice de Velocidade de Emergência de pau-ferro, com valores de 60,4\% e 0,330, respectivamente. A adubação química aumentou a produção de massa fresca e seca das plântulas, com valores de 399,9mg e 169,8mg, respectivamente, com médias que não variaram significativamente da maior dose de adubação orgânica. A adição de 19,46 g dm${ }^{-3}$ de adubo orgânico Organosuper® proporcionou maior número de plântulas, em menor tempo e com plântulas mais vigorosas.

Palavras-chave: Espécie nativa, Adubação orgânica e Mudas.
\end{abstract}

\section{GERMINATION AND GROWTH OF Caesalpinia ferrea MART. EX TUL. IN DIFFERENT SUBSTRATE}

\begin{abstract}
Seedlings production of native species for commercial planting and for recovery of degraded areas leads to an increasing seeking for technologies that reduce costs to establish native species, as for example alternative substratum. The objective of this work was to provide better emergency of seeds and initial growth of seedlings of Caesalpinia ferrea Mart. ex Tul. plants, therefore it was tested different types of substrates, using dystroferric red latosol, clay texture $-C$, mixed with sand $-S\left(0.5 \mathrm{dm}^{3}\right.$ of land $/ 0.5 \mathrm{dm}^{3}$ of sand), adding organic fertilizer (OF) organosuper ${ }^{\circledR}$ and chemical fertilizer (CF) Yoorin ${ }^{\circledR}$ at different ratios, constituting the following substrates: 1) $C+S$ (control); 2) $C+S+O F\left(9.86 \mathrm{~g} \mathrm{dm}^{-3}\right)$; 3) $\left.C+S+O F\left(14.69 \mathrm{~g} \mathrm{dm}^{-3}\right) ; 4\right) C+S+O F\left(19.46 \mathrm{~g} \mathrm{dm}^{-3}\right)$, 5) $S+C+C F\left(8.4 \mathrm{~g} \mathrm{dm}^{-3}\right)$. The composition earth + sand $+19.46 \mathrm{~g} \mathrm{dm}^{-3}$ of the organic fertilizer provided better emergence of seedlings and greater Emergence Speed Index of wood-iron seedlings, presenting values of 60.4 and 0.330 , respectively. Chemical fertilization increased the production of fresh and dry mass of seedlings, with values of 399.9mg and 169.8respectively, with means which did not significantly differ from the highest dose of organic fertilization.
\end{abstract}

Keywords: Native species, Organic fertilization and Seedlings.

\footnotetext{
${ }^{1}$ Recebido em 25.09.2008 e aceito para publicação em 18.04.2011.

${ }^{2}$ Universidade Federal da Grande Dourados, UFGD, Brasil. E-mail: <silvanascalon@ufgd.edu.br.>, <tiarakesli@yahoo.com.br> e<josenovelino@ufgd.edu.br>.

${ }^{3}$ Programa de Pós-Graduação em Ciências Biológicas pela Universidade Estadual Paulista Júlio de Mesquita Filho, UNESP, Brasil.E-mail: <bibakiss@yahoo.com.br>.

${ }^{4}$ Graduado em Agronomia pela Universidade Federal da Grande Dourados, UFGD, Brasil. E-mail: <leandromota@bol.com.br>.
} 


\section{INTRODUÇÃO}

O pau-ferro ou jucá pertence à família LeguminosaeCaesalpinoidae e é de fácil reconhecimento devido à presença de manchas claras no tronco, folíolos pequenos, flores amarelas e legumes duros (RIZZINI, 1995). É uma planta perenifólia ou semidecídua, de ampla dispersão e baixa densidade populacional, com importante uso na ornamentação, construção civil e marcenaria. Por ser uma espécie tolerante a áreas abertas, pode ser utilizada em programas de reflorestamento de áreas degradadas (LORENZI, 1992). Sua casca é muito usada na medicina popular, pois apresenta propriedades antiinflamatórias e analgésicas (CARVALHO et al., 1996). Devido ao cerne duro, a madeira dessa espécie tem sido utilizada para a confecção de vigas, esteios, estacas e lenha e, por essa razão, tem sido devastada, o que leva ao status de ameaçadas de extinção (LACERDA, 2001).

As sementes constituem a via de propagação mais empregada no estabelecimento da cultura, e a busca de conhecimentos sobre as condições ótimas para os testes de germinação desempenha papel fundamental dentro da pesquisa, além de fornecer informações valiosas sobre a propagação e potencialidades das espécies (VARELA et al., 2005). Os testes mais simples para a determinação de vigor são os de velocidade de desenvolvimento inicial, sendo os mais utilizados o tempo médio de germinação e o índice de velocidade de germinação, que se baseiam no pressuposto de que sementes mais vigorosas germinarão mais rapidamente do que outras em condições inferiores, distinguindo as sementes dentro do lote (PINÃ-RODRIGUES et al., 2004).

Para a comercialização de mudas, o viveirista busca a redução no tempo de permanência dessas no viveiro, consequentemente há redução do custo e aumento da qualidade. Visando à obtenção de mudas de qualidade, vários fatores são importantes, destacando-se o substrato utilizado, que deve proporcionar retenção de água suficiente para permitir a germinação e, quando saturado, deve manter quantidades adequadas de espaços porosos para facilitar o fornecimento de oxigênio, indispensável no processo de germinação (SMIDERLE;MINAME, 2001). A qualidade física do substrato é importante por ser utilizado num estádio de desenvolvimento em que a planta é muito suscetível ao ataque de microrganismos e pouco tolerante ao déficit hídrico.
Assim, o substrato deve reunir características físicas e químicas que promovam, respectivamente, a retenção de umidade e disponibilidade de nutrientes, de modo que atendam às necessidades da planta (CUNHA et al., 2006). Dessa forma, a escolha do substrato é de fundamental importância, pois é onde o sistema radicular irá se desenvolver, determinando o crescimento da parte aérea da muda (JABUR;MARTINS, 2002).

Os solos naturalmente férteis devem ser os preferidos para a semeadura, entretanto torna-se cada vez mais difícil encontrá-los, havendo assim a necessidade de fertilizá-los artificialmente. Atualmente, os fertilizantes orgânicos estão sendo bastante utilizados pelos viveiristas por atenderem as necessidades dos vegetais e também por serem de baixo custo e, sobretudo, por não serem poluentes e assim contribuir para a preservação do meio ambiente (BRASIL, 1999). Alguns adubos orgânicos são empregados em doses elevadas, e são pobres nos elementos nutritivos como nitrogênio, fósforo e potássio. Valem especialmente pela matéria orgânica, que, incorporada ao solo, se decompõe e forma húmus. Outros tipos de esterco, mais concentrados, comportam-se de modo mais semelhante ao dos adubos minerais, funcionando como fonte de nutrientes (MALAVOLTA et al., 2004). A adubação orgânica com cama de frango pode ser uma boa alternativa pela qualidade que possui de ser um adubo de liberação lenta, sofrendo menores perdas por lixiviação e disponibilizando por maior período seus nutrientes para as plantas. Souza et al. (2006a), avaliando o crescimento de diferentes espécies florestais, verificaram que as plantas cultivadas com adubação orgânica obtiveram os melhores desempenhos em todas as espécies, com destaque para substrato com esterco de frango.

Os adubos orgânicos proporcionam também maior capacidade de retenção de água, melhor estrutura, aeração e capacidade de ativar os processos microbianos (KHIEL, 1985; SILVAJÚNIOR; SIQUEIRA, 1997). Amatéria orgânica no solo auxilia também na regulação da temperatura, o que retarda a adsorção do fósforo mineral e fornece produtos da decomposição orgânica, que favorecem o desenvolvimento da planta (NOVAIS;SMITH, 1999). A utilização de adubação orgânica constituída a partir de matérias-prima (resíduos industriais e vegetais) e inoculada com um preparo à base de bactérias e enzimas (Extrato Biotecnológico Catalisador - HSNI) acelera

Revista Árvore, Viçosa-MG, v.35, n.3, Edição Especial, p.633-639, 2011 
o processo de compostagem e disponibiliza mais rapidamente macro e micronutrientes às plantas (HAYASHI;WATANABE, 2006).

A carência de estudos tem limitado o aumento na produção e qualidade das mudas de espécies nativas, pois são escassas as informações que indicam as dosagens e as fontes de fertilizantes mais adequados à obtenção de mudas de alta qualidade técnica e melhor adaptadas às condições de cada região. Baseado nesses fatos, este trabalho teve como objetivo avaliar a influência de fontes de adubação no substrato sobre a capacidade germinativa e o crescimento inicial de plântulas de pau-ferro.

\section{MATERIAL E MÉTODOS}

Este trabalho foi realizado em casa de vegetação da Universidade Federal da Grande Dourados-UFGD, com sementes de pau-ferro coletadas a partir de cinco matrizes, no Município de Dourados-MS, no período de julho a agosto de 2007. A cidade localiza-se a $22^{\circ} 13^{\prime} 16^{\prime \prime}$ de latitude sul e 5448’2' de longitude oeste, com altitude de 452 m, estado de Mato Grosso do Sul. O clima é classificado como Cwa e a precipitação média anual é de $1500 \mathrm{~mm}$, com temperatura média anual de $22^{\circ} \mathrm{C}$. Após exclusão de sementes chochas e danificadas, as restantes foram homogeneizadas e, em seguida, submetidas à escarificação química por imersão em ácido sulfúrico PA, por 20 minutos, agitando-se a solução com o auxílio de um bastão de vidro. Após a escarificação, procedeu-se à lavagem imediata das sementes em água corrente, durante $3 \mathrm{~min}$, e, em seguida, à secagem em temperatura ambiente de laboratório, sobre duas folhas de papel toalha.

Para semeadura, utilizou-se como substrato um Latossolo Vermelho Distroférrico, textura argilosa $\mathrm{T}$, misturado com areia $-\mathrm{A}\left(0,5 \mathrm{dm}^{3}\right.$ de terra $+0,5 \mathrm{dm}^{3}$ de areia), acrescentando diferentes proporções de adubo orgânico (AO) e adubo químico (AQ), constituindo os seguintes substratos: 1) $\mathrm{T}+\mathrm{A}$ (testemunha); 2) $\left.\left.\mathrm{A}+\mathrm{T}+\mathrm{AO}\left(9,86 \mathrm{~g} \mathrm{dm}^{-3}\right) ; 3\right) \mathrm{A}+\mathrm{T}+\mathrm{AO}\left(14,69 \mathrm{~g} \mathrm{dm}^{-3}\right) ; 4\right)$ $\mathrm{A}+\mathrm{T}+\mathrm{AO}\left(19,46 \mathrm{~g} \mathrm{dm}^{-3}\right)$ e de adubo orgânico composto por matérias-prima (resíduos industriais e animais, como restos de vísceras e sangue, e vegetais como cascas, talos e folhas, etc.) inoculadas com um preparo à base de bactérias e enzimas (Extrato Biotecnológico Catalizador - HSNI), conhecido como Organosusper ${ }^{\circledR}$ e adubo químico 5) $\mathrm{A}+\mathrm{T}+\mathrm{AQ}\left(8,4 \mathrm{~g} \mathrm{dm}^{-3}\right)$ do adubo químico (Termofosfato Yoorin), conhecido como Yoorin $\AA$.

A análise química dos diferentes substratos utilizados no estudo (Tabela 1) foi avaliada no Laboratório de Fertilidade de Solo da Faculdade de Ciências Agrárias/ UFGD. Os substratos foram colocados em bandejas de 128 células com aproximadamente $3 \times 3 \mathrm{~cm}$ de largura. A semeadura foi realizada na profundidade equivalente ao triplo do diâmetro das sementes, e as bandejas mantidas em casa de vegetação.

Foram avaliadas a porcentagem de emergência (\%E), o índice de velocidade de emergência (IVE). A cada onze dias, foram coletadas duas plântula de cada substrato e avaliadas quanto à altura e ao comprimento radicular, e massa fresca e seca da plântula.

Para avaliação da Emergência e do Índice de Velocidade de Emergência, o delineamento estatístico adotado foi o inteiramente casualizado com cinco tratamentos, e, para as demais características, delineamento inteiramente casualizado em esquema fatorial ,com cinco substratos e quatro períodos de avaliação, ambos com quatro repetições de 20 sementes. Os dados foram submetidos à análise de variância, havendo significância. As médias de substrato foram

Tabela 1 - Emergência (E), índice de velocidade de emergência (IVE), altura, comprimento de raiz (CR), massa fresca (MF) e seca (MS) de plantas de Caesalpinia ferrea Mart. ex Tul aos 53 dias após a semeadura. UFGD, 2010.

Table 1 -Emergency (E), emergence speed index (ESI), height, root length (RL), fresh (FM) and dry mass (DM) of Caesalpinia ferrea Mart. ex Tu, seedlings on the $53^{\text {rdh }}$ day after sowing. UFGD, 2010.

\begin{tabular}{lcccccc}
\hline Substratos & E(\%) & IVE & A(cm) & CR(cm) & MF(mg) & MS(mg) \\
\hline T+A & $45,8 \mathrm{~b}$ & $0,255 \mathrm{~b}$ & $11,5 \mathrm{~b}$ & $9,8 \mathrm{a}$ & $375,3 \mathrm{c}$ & $140,1 \mathrm{c}$ \\
T+A+AO $\left(9,86 \mathrm{~g} \mathrm{dm}^{-3}\right)$ & $41,6 \mathrm{~b}$ & $0,238 \mathrm{~b}$ & $11,4 \mathrm{~b}$ & $9,4 \mathrm{~b}$ & $359,5 \mathrm{c}$ & $141,3 \mathrm{c}$ \\
$\mathrm{T}+\mathrm{A}+\mathrm{AO}\left(14,69 \mathrm{~g} \mathrm{dm}^{-3}\right)$ & $49,9 \mathrm{~b}$ & $0,305 \mathrm{~b}$ & $12,0 \mathrm{a}$ & $9,2 \mathrm{~b}$ & $388,1 \mathrm{~b}$ & $149,9 \mathrm{~b}$ \\
T+A+AO $\left(19,46 \mathrm{~g} \mathrm{dm}^{-3}\right)$ & $60,4 \mathrm{a}$ & $0,330 \mathrm{a}$ & $12,3 \mathrm{a}$ & $9,1 \mathrm{~b}$ & $390,0 \mathrm{a}$ & $156,7 \mathrm{a}$ \\
T+A+AQ $\left(8,4 \mathrm{~g} \mathrm{dm}^{-3}\right)$ & $41,7 \mathrm{~b}$ & $0,263 \mathrm{~b}$ & $11,8 \mathrm{~b}$ & $9,1 \mathrm{~b}$ & $399,9 \mathrm{a}$ & $169,8 \mathrm{a}$ \\
CV(\%) & $31,7 \%$ & $30,9 \%$ & $8,6 \%$ & $14,3 \%$ & $16,2 \%$ & $21,3 \%$ \\
\hline
\end{tabular}

Médias seguidas de mesma letra são estatisticamente iguais pelo teste de Duncan a 5\% de probabilidade.

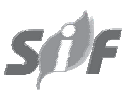

Revista Árvore, Viçosa-MG, v.35, n.3, Edição Especial, p.633-639, 2011 
comparadas pelo teste de Duncan, a 5\%, e de períodos de avaliação por análise de regressão utilizando o programa SANEST.

\section{RESULTADOS E DISCUSSÃO}

A porcentagem de emergência e índice de velocidade de emergência foram maiores quando a semeadura ocorreu no substrato 4, com maior quantidade de matéria orgânica (19,46 g dm ${ }^{-3}$ de adubo orgânico organosuper $\left.®\right)$.Aemergência iniciou-se aos 9 dias após a semeadura, chegando ao final do período de avaliação com 60,4\% (Tabela 1). De acordo com a análise química dos substratos (Tabela 2), aqueles com menores quantidades de matéria orgânica apresentaram menor porcentagem de emergência e índice de velocidade de emergência. Esses resultados provavelmente devem estar relacionados a menores taxas de retenção de água e baixa porosidade desses substratos. Souza et al. (2001), verificaram no substrato com maior quantidade de matéria orgânica (terriço de mata + vermiculita) uma emergência mais rápida para plantas de cagaita (Eugenia dysenterica DC) em comparação aos demais substratos. O aumento na germinação em espécies florestais, ao se adicionar húmus de minhoca como adubação orgânica, foi observado por Berton (1999).

Os substratos 3 e 4 contendo $14,69 \mathrm{~g} \mathrm{dm}^{-3} \mathrm{e}$ 19,46 $\mathrm{g} \mathrm{dm}^{-3}$ do adubo orgânico organosuper ${ }^{\circledR}$, respectivamente, apresentaram maiores valores de $\mathrm{K}$, Ca e Mg em relação aos demais substratos (Tabela1), provavelmente esses macronutrientes tenham favorecido os melhores resultados para o crescimento da parte aérea, quando comparados com os substratos sem adubação, com menor adição de adubo orgânico ou adubação química (Tabela 2). Cunha et al. (2006), avaliando o desenvolvimento de mudas de Acácia sp em diferentes substratos, verificaram, na parte aérea das mudas, tendência ao maior acúmulo de $\mathrm{P}, \mathrm{K}$ e Mg e maior altura quando cultivadas no substrato com adição de esterco bovino, revelando nesse substrato quantidades relevantes desses nutrientes. Gomes et al. (2002), avaliando os parâmetros morfológicos de mudas de Eucaliptus grandis, consideram a altura da parte aérea um atributo muito importante para estimar o padrão de qualidade de mudas.

Não houve interação significativa entre os diferentes substratos e épocas de avaliação para nenhuma das características avaliadas. Em relação ao crescimento do sistema radicular, verificou-se no substrato 1 (terra+areia) maior comprimento médio $(9,8 \mathrm{~cm})$. Este fato pode ter ocorrido em função da baixa disponibilidade de nutrientes e água neste substrato, forçando as raízes a um maior crescimento axial em busca de nutrientes. A mesma tendência foi observada por Campos et al. (1986) ao avaliarem o desenvolvimento inicial de sibipiruna (Caesalpinia peltophoroides Benth), verificando no substrato sem adubação (terra+areia) maior comprimento de raiz principal $(33,27 \mathrm{~cm})$, observados aos 212 dias após a semeadura.

Já para a produção de massa fresca e seca das plântulas de pau-ferro, o substrato 4 e 5, contendo a maior dose de adubo orgânico ou adubo químico Yoorin ${ }^{\circledR}$, proporcionaram os maiores valores, que não variaram estatisticamente entre si. Entretanto, pela análise química desse substrato, foi observada menor quantidade de nutrientes quando comparado com a adubação

Tabela 2 - Análise química dos substratos utilizados no experimento. UFGD, 2010.

Table 2 - Chemical analysis of substrates used in the experiment. UFGD, 2010.

\begin{tabular}{|c|c|c|c|c|c|}
\hline \multirow[t]{2}{*}{ Atributos Químicos } & \multicolumn{5}{|c|}{$\mathrm{g} \mathrm{dm}^{-3}$} \\
\hline & $\mathrm{T}+\mathrm{A}$ & $\mathrm{T}+\mathrm{A}+\mathrm{AO}\left(9,86^{1}\right)$ & $\mathrm{T}+\mathrm{A}+\mathrm{AO}\left(14,69^{1}\right)$ & $\mathrm{T}+\mathrm{A}+\mathrm{AO}\left(19,46^{1}\right)$ & $\mathrm{T}+\mathrm{A}+\mathrm{AQ}\left(8,4^{2}\right)$ \\
\hline$\overline{\text { M.O. }\left(\mathrm{g} \mathrm{kg}^{-1}\right)}$ & 2,6 & 4,5 & 4,8 & 6,1 & 4,5 \\
\hline $\mathrm{PH}$ em $\mathrm{H}_{2} \mathrm{O}-1: 2,5$ & 5,3 & 5,6 & 6,4 & 6,6 & 5,6 \\
\hline $\mathrm{P}-$ Melilich $\left(\mathrm{mg} \mathrm{dm}^{-3}\right)$ & 15 & 77 & 96 & 187 & 113 \\
\hline $\mathrm{K}-$ Melilich (mg) dm ${ }^{-3}$ ) & 1,5 & 5,5 & 7,4 & 8,7 & 0,8 \\
\hline Al - Trocável (mmol $\left.\mathrm{dm}^{-3}\right)$ & 0,0 & 0,0 & 0,0 & 0,0 & 9,8 \\
\hline Ca- Trocável (mmol $\mathrm{dm}^{-3}$ ) & 14 & 20 & 24 & 29 & 8 \\
\hline Mg- Trocável (mmol dm²) & 2 & 12 & 16 & 20 & 5 \\
\hline $\mathrm{H}+\mathrm{Al}-\left(\mathrm{mmol} \mathrm{dm}^{-3}\right)$ & 25 & 24 & 17 & 16 & 45 \\
\hline S.Bases $-\left(\mathrm{mmol} \mathrm{dm}^{-3}\right)$ & 17,5 & 37,5 & 47,4 & 57,4 & 13,8 \\
\hline $\mathrm{T}$ - CTC Total (mmol $\left.\mathrm{dm}^{-3}\right)$ & 42,5 & 61,5 & 64,4 & 73,4 & 58,8 \\
\hline V - Sat. Bases (\%) & 41 & 60 & 73 & 78 & 23 \\
\hline
\end{tabular}

1 e ${ }^{2}$ Respectivamente, massas de adubo orgânico e químico adicionadas a misturas de 0,5 dm³ de areia - A e 0,5 dm³ de solo - T.

Revista Árvore, Viçosa-MG, v.35, n.3, Edição Especial, p.633-639, 2011 
orgânica. Souza et al. (2001), avaliando a adição de adubação química (Termofosfato Yoorin) no substrato, observaram resultados superiores de massa fresca e seca para as plântulas de cagaita (Eugenia dysenterica DC), embora, pela análise química, este substrato tenha também apresentado menores teores de nutrientes em relação aos demais. Esses autores sugerem que, por se tratar de uma espécie que ocorre em solos pobres de cerrado, o aumento na fertilidade é benéfico até um limite a partir do qual a adubação química passa a agir de forma negativa sobre o crescimento dessa espécie. Embora as espécies dos cerrados sejam adaptadas a solos de baixa fertilidade, pesquisas têm demonstrado efeitos benéficos de adubação. Souza et al. (2006b), avaliando o desenvolvimento inicial de mudas de ipêroxo (Tabebuia impertiginosa Mart. ex D.C.), verificaram que estas respondem positivamente à adição de nutrientes, quando semeada em Latossolo VermelhoAmarelo de baixa fertilidade.

a)

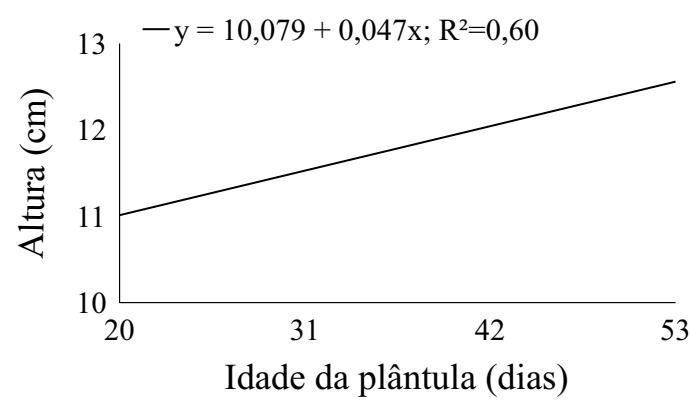

c)

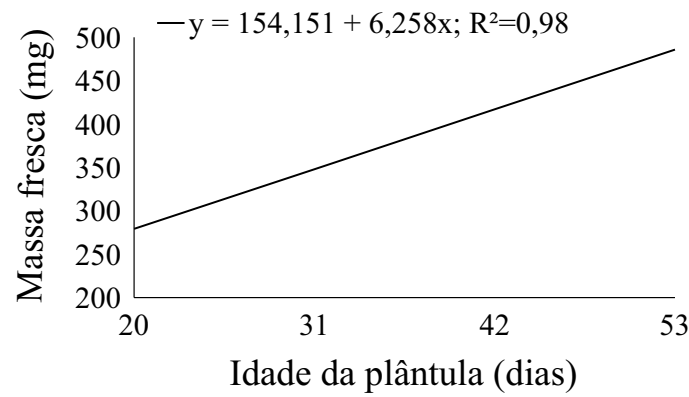

O teor de alumínio no substrato 5 não prejudicou o crescimento das mudas, embora se encontra um pouco acima daquele observado em solo natural de cerrado, com média em torno de 6,1 mmol dm³ (SANGALLI, 2008).

Houve crescimento linear na altura e no comprimento de raiz e das massas fresca e seca por dia, em média, 0,047 cm de altura; 0,05 cm de raiz; 6,258mg de massa fresca e 2,669mg de massa seca. Em relação à altura e ao comprimento de raiz, observaram-se, no final das avaliações, valores médios de 12,56 cm e 10,13 cm, respectivamente (Figura 1). Já para massa fresca e seca, as plântulas apresentaram 485,8 mg e 195,6 mg, respectivamente. Nietshe et al. (2004), ao avaliarem o crescimento inicial de plantas de cagaiteira, concluíram que apresentam crescimento lento (5,6 cm de altura) verificado aos 182 dias após a semeadura. Os autores atribuem esse lento crescimento a fatores genéticos ou à baixa concentração de nutrientes e matéria orgânica

b)

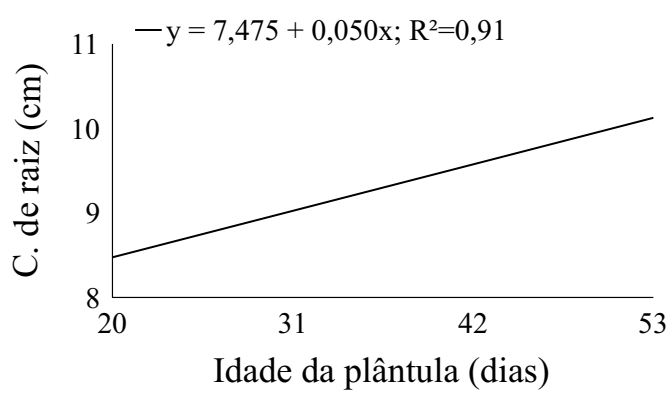

d)

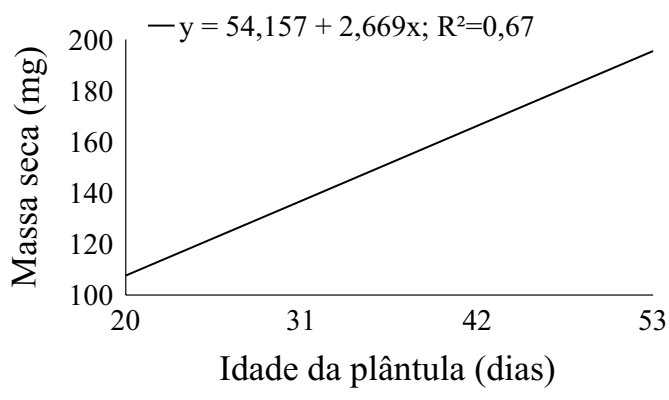

Figura 1- Altura, comprimento de raiz, massa fresca e seca de plântulas de Caesalpinea ferrea Mart. ex Tul em função da idade. UFGD, 2010.

Figure 1 - Height, root length, and dry mass of Caesalpinea ferrea Mart. ex Tul seedlings according to their age. UFGD, 2010. 
nos substratos utilizados. Baseado nessas informações, pode-se considerar que o pau-ferro apresentou crescimento satisfatório e é uma espécie que responde ao fornecimento de nutrientes, assim como observado por Souza et al. (2006b), para mudas de ipê-roxo.

\section{CONCLUSÃO}

A adição de 19,46 g dm$^{-3}$ de adubo orgânico Organosuper® proporciona maior número de plântulas, em menor tempo, e plântulas mais vigorosas.

\section{REFERÊNCIAS}

BERTON, R. S. Agricultura orgânica.

Campinas: Instituto Agronômico de Campinas, 1999.

BRASIL. Ministério do Meio ambiente Lei no 9.605, de 12 de fevereiro de 1998. Lei dos crimes ambientais. Brasília: 1999. 38p.

CAMPOS, L. A. A. et al. Influência de profundidade de semeadura e substratos no desenvolvimento inicial de sibipiruna (Caesalpinia peltophoroides BENTH), Científica, v.14, n. 1/2, p.101-113, 1986.

CARVALHO, J. C. T. et al. Preliminary studies of analgesic and anti-inflammatory properties of Caesalpinea ferrea crude extract, Journal of Ethnopharmacology, v.53, p.175-178, 1996.

CUNHA, A. M. et al. Efeito de diferentes substratos sobre o desenvolvimento de mudas de Acacia sp. Revista Árvore, v.30, n.1, p.207-214, 2006.

GOMES, J. M. et al. Parâmetros morfológicos na avaliação da qualidade de mudas de Eucaliptus grandis. Revista Árvore, v.26, n.6, p.655-664, 2002.

HAYASHI, F.; WATANABE, S. Registro de estabelecimento e produto. Brasilia Ministério da Agricultura, Pecuária e Abastecimento, 2006.

JABUR, M. A.; MARTINS, A. B. G. Influência de substratos na formação dos porta-enxertos: limoeirocravo (Citrus Limonia Osbeck) e tangerineiracleópatra (Citrus Reshni Hort. ex Tanaka) em ambiente protegido. Revista Brasileira de Fruticultura, v.24, n.2, p.514-518, 2002.
KIEHL, E. J. Fertilizantes orgânicos. Piracicaba: Agronômica Ceres, 1985. 492p.

LACERDA, S. N. Estudo de diferentes técnicas de armazenagem para conservação de sementes nativas do semi-árido paraibano. 2001. 88f Dissertação (Mestrado em Engenharia Agrícola) Universidade Federal da Paraíba, Campina Grande, 2001. 88p.

LORENZI, H. Árvores brasileiras: manual de identificação e cultivo de plantas arbóreas nativas do Brasil. Nova Odessa: Plantarum, 1992. 365p.

MALAVOLTA, E.; GOMES, P. F.; ALCARDE, J. C. Adubos e adubações. São Paulo: Nobel, 2004. 1970p.

NIETSHE, S. et al. Tamanho da semente e substratos na germinação e crescimento inicial de mudas de cagaiteira. Ciência Agrotécnica, v.28, n.6, p.1321-1325, 2004.

NOVAIS, R.; SMYTH, T. J. Fósforo em solo e planta em condições tropicais. Viçosa, MG: Universidade Federal de Viçosa, 1999. 339p.

PINÃ-RODRIGUES, F. C. M.; FILGLIOLIA, M. B.; PEIXOTO, M. C. Testes de qualidade. In: FERREIRA, A. G.; BORGUETTI, F. (Orgs.). Germinação - do básico ao aplicado. Porto Alegre: Artmed, 2004. p.283-297.

RIZZINI, C. T. Botânica econômica brasileira. 2.ed. Rio de Janeiro: Âmbito Cultural, 1995. 248p.

SANGALLI, A. Morfometria, crescimento e produção de Jacarandá decurrens summetrifoliolata FARIAS \& PROENÇA. 2008. 180f. Tese (Doutorado em Agronomia) Universidade Federal da Grande Dourados, Dourados, 2008.

SILVA JUNIOR, J. P.; SIQUEIRA, J. O. Aplicação de formononetina sintética ao solo como estimulante da formação de micorriza no milho e na soja. Revista Brasileira de Fisiologia Vegetal, v.9, n.1, p.35-41, 1997.

SMIDERLE, O. S.; MINAME, K. Emergência e vigor de plântulas de goiabeira em diferentes substratos. Revista Científica Rural, n.6, n.1, p.38-45, 2001. 
SOUZA, E. R. B. et al. Emergência e crescimento de cagaita (Eugenia dysenterica DC.) em função do tipo e volume de substratos. Pesquisa Agropecuária Tropical, v.31, n.2, p.89-95, 2001.

SOUZA, C.A.M. et al. Crescimento em campo de espécies florestais em diferentes condições de adubações. Ciência Florestal, v.16, n.3, p.243-249, 2006a.
SOUZA, P. A.; VENTURIN, N.; MACEDO, R. L. G. Adubação mineral do ipê-roxo (Tabebuia impetiginosa). Ciência Florestal, v.16, n.3, p.261-270, 2006b

VARELA,V. P.; COSTA, S. S.; RAMOS, M. B. P. Influência da temperatura e do substrato na germinação de sementes de itaubarana (Acosmium nitens (Vog.) Yakovlev) - Leguminosae,

Caesalpinoideae. Acta Amazonica, v.35, n.1, p.35-39, 2005. 
